\title{
INJECTIVE AND PROJECTIVE HEYTING ALGEBRAS $\left({ }^{1}\right)$
}

\author{
BY

\section{RAYMOND BALBES AND ALFRED HORN}

The determination of the injective and projective members of a category is usually a challenging problem and adds to knowledge of the category. In this paper we consider these questions for the category of Heyting algebras. There has been a lack of uniformity in terminology in recent years. In [6] Heyting algebras are referred to as pseudo-Boolean algebras, and in [1] they are called Brouwerian lattices. We would argue for retaining the name Heyting algebras for the reasons given in [2, p. 162j, reserving the name Brouwerian algebras for the algebras dual to Heyting algebras. The fundamental paper on Brouwerian algebras (and therefore Heyting algebras) is [4].

We shall show that a Heyting algebra is injective if and only if it is a complete Boolean algebra. The determination of projective Heyting algebras is, as usual, more difficult. We shall characterize all projective Heyting algebras which are finite or are chains. Some other results on projective algebras are given.

1. Definitions and preliminaries. If $S$ is a subset of a lattice $L$, then $\Sigma(S)$ denotes the least upper bound in $L$ of $S$, and $\Pi(S)$ denotes the greatest lower bound of $S$. If $S=\{x, y\}$, then $\Sigma(S)$ and $\Pi(S)$ are written $x+y$ and $x y$. The largest and smallest elements of a lattice $L$ are denoted by 1 (or $1_{L}$ ) and 0 respectively.

A Heyting algebra is a lattice with 0 such that for any elements $a, b$ there is an element $a \rightarrow b$ such that $a x \leqq b$ if and only if $x \leqq a \rightarrow b$. We use $\neg a$ to denote $a \rightarrow 0$. Every Heyting algebra is a distributive lattice. The lattice of all open sets of a topological space is a Heyting algebra. We state without proof some elementary properties of Heyting algebras.

THEOREM 1.1. If $a, b$, and $c$ are any elements of a Heyting algebra, then the following hold:

(1) $a \leqq b$ if and only if $a \rightarrow b=1$.

(2) $1 \rightarrow a=a$.

(3) $a \rightarrow b \geqq b$.

(4) If $b \leqq c$, then $a \rightarrow b \leqq a \rightarrow c$ and $c \rightarrow a \leqq b \rightarrow a$.

Received by the editors October 4, 1968.

(1) The research and preparation of this paper was supported in part by NSF GP-9044 and by an assistant professor research fund grant from the University of Missouri-St. Louis.

Copyright (C) 1970, American Mathematical Society 
(5) $a \rightarrow(b \rightarrow c)=a b \rightarrow c$.

(6) If $a \leqq c$ and $a \rightarrow b=b$, then $c \rightarrow b=b$.

(7) $(a+b) \rightarrow c=(a \rightarrow c)(b \rightarrow c)$.

(8) $a \leqq(a \rightarrow b) \rightarrow b$.

(9) $((a \rightarrow b) \rightarrow b) \rightarrow b=a \rightarrow b$.

(10) $((a \rightarrow b) \rightarrow b) \rightarrow(a \rightarrow b)=a \rightarrow b$.

(11) $(a \rightarrow b) \rightarrow((a \rightarrow b) \rightarrow b)=(a \rightarrow b) \rightarrow b$.

(12) If $a \leqq b, a(b \rightarrow c)=a c$.

From the point of view of universal algebra, Heyting algebras are regarded as algebras $\langle H,+, \cdot, \rightarrow, 0\rangle$ with three binary operations and a distinguished element. Accordingly subalgebras and homomorphisms of Heyting algebras are subsets and functions which preserve the operations and 0 . A Heyting homomorphism $f$ is one-to-one (that is, $f$ is a monomorphism) if and only if $f(u)=1$ only for $u=1$. We shall usually refer to Heyting homomorphisms by using the word homomorphism without qualification. A Heyting algebra $H$ is said to be injective (in the category of Heyting algebras) if for any Heyting monomorphism $f: B \rightarrow A$ and any homomorphism $h: B \rightarrow H$, there exists a homomorphism $g: A \rightarrow H$ such that $g f=h$. A Heyting algebra $H$ is called projective if for any Heyting epimorphism $f: A \rightarrow B$ and any homomorphism $h: H \rightarrow B$, there exists a homomorphism $g: H \rightarrow A$ such that $f g=h$.

The identity map of a set $A$ is denoted by $I_{A}$. A Heyting algebra $H$ is a retract of an algebra $K$ if there exist homomorphisms $f: K \rightarrow H$ and $g: H \rightarrow K$ such that $f g=I_{H}$. A retract of a projective Heyting algebra is projective. A Heyting algebra is projective if and only if it is a retract of a free Heyting algebra. If $f: K \rightarrow H$ is a Heyting epimorphism and $H$ is projective, then $H$ is a retract of $K$.

Boolean algebras will be regarded as algebras $\left\langle B,+, \cdot,{ }^{-}\right\rangle$where $\bar{a}$ denotes the complement of $a$. There are certain connections between Heyting algebras and Boolean algebras which we shall use. If $\left\langle B,+, \cdot,^{-}\right\rangle$is a Boolean algebra, then $\left\langle B,+, \cdot, \rightarrow, 0_{B}\right\rangle$ is a Heyting algebra, where $a \rightarrow b=\bar{a}+b$. If $B_{1}$ and $B_{2}$ are Boolean algebras then a map $f: B_{1} \rightarrow B_{2}$ is a Heyting homomorphism if and only if it is a Boolean homomorphism. If $H$ is a Heyting algebra, let $R(H)$ be the set of regular elements, that is, $R(H)=\{x \in H: x=\neg \neg x\}$. The following theorem is well known.

THEOREM 1.2. If $H$ is a Heyting algebra, then $\langle R(H), \dot{+}, \cdot, \neg\rangle$ is a Boolean algebra, where $a+b=\neg \neg(a+b)$. The canonical map $\varphi: H \rightarrow R(H)$ defined by $\varphi(x)=\neg \neg x$ is a Heyting epimorphism.

If $H$ is a Heyting algebra, then $\langle H,+, \cdot, \neg\rangle$ is a Boolean algebra if and only if $\neg x=0$ only for $x=1$. This follows immediately from the identity $\neg(x+\neg x)=0$.

A linearly ordered set with 0 and 1 will be called a chain. Every chain is a Heyting algebra in which $x \rightarrow y=y$ for $x>y$ and $x \rightarrow y=1$ for $x \leqq y$. By a subchain of a Heyting algebra, we mean a subalgebra which is a chain. 
If $L$ is a lattice and $x \in L$, let $L_{(x)}$ denote $\{y \in L: y \leqq x\}$ and $L^{(x)}$ denote $\{y \in L: y \geqq x\}$.

\section{Injective Heyting algebras.}

LEMMA 2.1. Let $f: H \rightarrow K$ and $g: K \rightarrow H$ be order-preserving maps of partially ordered sets such that $g f=I_{H}$. If $K$ is complete, then $H$ is complete.

Proof. Let $\left\{x_{\alpha}: \alpha \in I\right\}$ be any family of elements of $H$. Let $y=\Sigma\left\{f\left(x_{\alpha}\right): \alpha \in I\right\}$. Then $g(y) \geqq g f\left(x_{\alpha}\right)=x_{\alpha}$ for all $\alpha \in I$. If $z \in H$ and $z \geqq x_{\alpha}$ for all $\alpha$, then $f(z) \geqq f\left(x_{\alpha}\right)$ for all $\alpha$. Therefore $f(z) \geqq y$ and $z=g f(z) \geqq g(y)$. Hence $g(y)=\Sigma\left\{x_{\alpha}: \alpha \in I\right\}$.

THEOREM 2.2. If $H$ is an injective Heyting algebra, then $H$ is a complete Boolean algebra.

Proof. Let $K$ be the set of all ideals of $H$. Then $K$ is a complete Heyting algebra [1, p. 129]. For $x \in H$, let $f(x)=H_{(x)}$. Then $f: H \rightarrow K$ is a monomorphism. Since $H$ is injective, there exists a homomorphism $g: K \rightarrow H$ such that $g f=I_{H}$. By 2.1, $H$ is complete.

To prove $H$ is a Boolean algebra, we must show $\neg x=0$ only for $x=1$. Suppose there exists $a \in H$ such that $\neg a=0$ and $a<1$. Then $\{0, a, 1\}$ is a subchain of $H$. Extend it to a maximal subchain $C$ of $H$. Let $g: C \rightarrow H$ be defined by $g(x)=x$. Let $D$ be the chain obtained by adding to $C$ a new element $\alpha$ such that $0<\alpha<x$ for all $x \in C-\{0\}$. Then $C$ is a subchain of $D$. Since $H$ is injective, there exists a homomorphism $h: D \rightarrow H$ which extends $g$. Now $h(D)$ is a subchain of $H$ containing $C$. By the maximality of $C, h(\alpha) \in C$. But $h(\alpha) \neq 0$, since $\neg h(\alpha)=h(\neg \alpha)=h(0)=0$. Also $h(\alpha) \leqq h(a)=a<1$. If $x \in C-\{0\}$, then $h(\alpha) \leqq x$ and $x \rightarrow h(\alpha)=h(x \rightarrow \alpha)$ $=h(\alpha)<1$. Therefore $h(\alpha)<x$ for all $x \in C-\{0\}$ by $1.1(1)$. Thus $h(\alpha) \notin C$ and we have a contradiction.

Lemma 2.3. Let $h: H \rightarrow B$ be a Heyting homomorphism from a Heyting algebra $H$ to a Boolean algebra $B$. Then there exists a Boolean homomorphism $h_{1}: R(H) \rightarrow B$ such that $h_{1} \varphi=h$, where $\varphi: H \rightarrow R(H)$ is the canonical map of 1.2.

Proof. Since $h(\neg x)=\overline{h(x)}$, we have $h(\neg \neg x)=h(x)$ for all $x \in H$. Let $h_{1}$ be the restriction of $h$ to $R(H)$. If $x, y \in R(H), h(x+y)=h(\neg \neg(x+y))=h(x+y)=h_{1}(x)$ $+h_{1}(y)$. Therefore $h_{1}$ is a Boolean homomorphism. Also $h_{1} \varphi(x)=h(\neg \neg x)=h(x)$ for all $x \in H$.

Lemma 2.4. Let $f: H_{1} \rightarrow H_{2}$ be a Heyting homomorphism. Then there exists a Boolean homomorphism $f^{\prime}: R\left(H_{1}\right) \rightarrow R\left(H_{2}\right)$ such that $\varphi_{2} f=f^{\prime} \varphi_{1}$, where $\varphi_{1}, \varphi_{2}$ are the canonical maps of 1.2. If $f$ is one-to-one, so is $f^{\prime}$.

Proof. The existence of $f^{\prime}$ follows immediately from 2.3. Assume $f$ is one-to-one. Suppose $x \in R\left(H_{1}\right)$ and $f^{\prime}(x)=1$. Then $1=f^{\prime}(x)=f^{\prime}(\neg \neg x)=f^{\prime} \varphi_{1}(x)=\varphi_{2} f(x)$ $=\neg \neg f(x)=f(\neg \neg x)$. Therefore $\neg \neg x=1$ and so $x=1$. Hence $f^{\prime}$ is one-to-one. 
THEOREM 2.5. A Heyting algebra $H$ is injective if and only if it is a complete Boolean algebra.

Proof. The necessity was proved in 2.2. Suppose $H$ is a complete Boolean algebra. Let $f: H_{1} \rightarrow H_{2}$ be a Heyting monomorphism, and let $h: H_{1} \rightarrow H$ be a Heyting homomorphism. Let $\varphi_{i}: H_{i} \rightarrow R\left(H_{i}\right)$ be the canonical maps, $i=1,2$. By 2.3 there exists a Boolean homomorphism $h_{1}: R\left(H_{1}\right) \rightarrow H$ such that $h_{1} \varphi_{1}=h$. By 2.4 there exists a Boolean monomorphism $f^{\prime}: R\left(H_{1}\right) \rightarrow R\left(H_{2}\right)$ such that $\varphi_{2} f=f^{\prime} \varphi_{1}$. Now Sikorski's theorem implies that $H$ is injective in the category of Boolean algebras [3, p. 141]. Therefore there exists a Boolean homomorphism $g_{1}: R\left(H_{2}\right) \rightarrow H$ such that $g_{1} f^{\prime}=h_{1}$. Let $g=g_{1} \varphi_{2}: H_{2} \rightarrow H$. Then $g f=g_{1} \varphi_{2} f=g_{1} f^{\prime} \varphi_{1}$ $=h_{1} \varphi_{1}=h$.

\section{Star sums of Heyting algebras.}

Definition 3.1. If $L$ is a lattice, then $L \oplus 1$ denotes the lattice obtained by adding a new element 1 such that $1>x$ for all $x \in L$.

Definition 3.2. A pre-Heyting algebra is a lattice $L$ such that $x \rightarrow y$ exists whenever $x, y \in L$ and $x \neq y$.

Definition 3.3. A pre-subalgebra of a pre-Heyting algebra $L$ is a sublattice $S$ such that $x \rightarrow y \in S$ whenever $x, y \in S$ and $x \neq y$.

If $L$ is a pre-Heyting algebra with 0 and 1 , then $L$ is a Heyting algebra by 1.1 (1). If $L$ is a pre-Heyting algebra with 0 , then $L \oplus 1$ is a Heyting algebra and $L$ is a pre-subalgebra of $L \oplus 1$.

Definition 3.4. If $L_{1}$ is a pre-Heyting algebra and $L_{2}$ is a Heyting algebra, a function $f: L_{1} \rightarrow L_{2}$ is called a pre-homomorphism if $f$ is a lattice homomorphism and $f(x \rightarrow y)=f(x) \rightarrow f(y)$ whenever $x$

Definition 3.5. An element $\alpha$ of a lattice $L$ is called a node if $\alpha$ is comparable with every element of $L$.

THEOREM 3.6. If $\alpha$ is an element of a Heyting algebra $H$, then $\left\langle H^{(\alpha)},+, \cdot, \rightarrow, \alpha\right\rangle$ is a Heyting algebra. If $\alpha$ is a node, then $H_{(\alpha)}$ is a Heyting algebra which is a presubalgebra of $H$.

Proof. The proof is easy and is omitted.

Definition 3.7. Suppose $\delta$ is an ordinal such that $0<\delta \leqq \omega$, and $H_{n}$ is a Heyting algebra for each $n, 0 \leqq n<\delta$. By $\Sigma_{n<\delta}^{*} H_{n}$ we mean a lattice $H$ with nodes $0_{H}=\alpha_{0}$ $<\alpha_{1}<\cdots<\alpha_{\delta}=1_{H}$ such that for each $n, H_{n}$ is isomorphic with

$$
H_{n}^{\prime}=\left\{x \in H: \alpha_{n} \leqq x \leqq \alpha_{n+1}\right\} .
$$

We will usually identify $H_{n}$ with $H_{n}^{\prime}$, so that $\alpha_{n}=0_{H_{n}}$ and $\alpha_{n+1}=1_{H_{n}}$. If $\delta$ is finite, then $\Sigma_{n<\delta}^{*} H_{n}$ is written $H_{0} * H_{1} * \cdots * H_{\delta-1}$.

THEOREM 3.8. If $H=\Sigma_{n<\delta}^{*} H_{n}$, then $H$ is a pre-Heyting algebra, and for each $n$, $H_{n}$ is a pre-subalgebra of $H$. If $\delta$ is finite, $H$ is a Heyting algebra. 
Proof. Suppose $x \in H_{n}, y \in H_{m}$ and $x \leq y$. It is easy to verify that if $m=n$, then $x \rightarrow y$ has the same meaning in $H$ as in $H_{n}$, while if $m<n$ and $y<0_{H_{n}}$ then $x \rightarrow y=y$.

Lemma 3.9. Suppose $f_{0}: H_{0} \rightarrow K_{0}$ is a Heyting isomorphism and $f_{1}: H_{1} \rightarrow K_{1}$ is a Heyting homomorphism. Then the function $g: H_{0} * H_{1} \rightarrow K_{0} * K_{1}$ defined by $g(x)=f_{i}(x)$ for $x \in H_{i}, 1=1,2$, is a Heyting homomorphism.

Proof. This follows easily from 3.8 and its proof.

Definition 3.10. Let $B_{2}$ and $B_{4}$ denote the Boolean algebras with two and four elements respectively.

Observe that if $H$ is a Heyting algebra, then $H \oplus 1=H * B_{2}$.

Lemma 3.11. Let $H$ be a Heyting algebra with a node $\alpha$ such that $H^{(\alpha)}=\left\{\alpha, 1_{H}\right\}$, and let $F$ be a Heyting algebra. Suppose $g: H_{(\alpha)} \rightarrow F$ is a pre-homomorphism, and $x$ is any member of $F$. Let $h: H \rightarrow F$ be the extension of $g$ such that $h\left(1_{H}\right)=x+$ $(x \rightarrow g(\alpha))$. Then $h$ is a pre-homomorphism.

Proof. $h$ is obviously a lattice homomorphism, since $h\left(1_{H}\right) \geqq g(\alpha)$ by $1.1(3)$. If $u \in H_{(\alpha)}$ we have

$$
\begin{aligned}
h\left(1_{H}\right) \rightarrow h(u) & =(x+(x \rightarrow g(\alpha))) \rightarrow g(u) \\
& =(x \rightarrow g(u)) \cdot((x \rightarrow g(\alpha)) \rightarrow g(u)) \quad \text { by } 1.1(7) \\
& =(x \rightarrow g(u)) \cdot g(u) \quad \text { by } 1.1(4) \text { and }(12) \\
& =g(u) \quad \text { by } 1.1(3) \\
& =h(u)=h\left(1_{H} \rightarrow u\right) .
\end{aligned}
$$

Lemma 3.12. Let $H$ be a Heyting algebra with a node $\alpha$ such that $H^{(\alpha)}=\left\{\alpha, \beta, \gamma, 1_{H}\right\}$ is isomorphic with $B_{4}$. Let $F$ be a Heyting algebra, let $g: H_{(\alpha)} \rightarrow F$ be a pre-homomorphism, and let $y$ be any member of $F$. Let $h: H \rightarrow F$ be the extension of $g$ such that $h(\beta)=(y \rightarrow g(\alpha)) \rightarrow g(\alpha), h(\gamma)=y \rightarrow g(\alpha)$, and $h\left(1_{H}\right)=h(\beta)+h(\gamma)$. Then $h$ is $a$ pre-homomorphism.

Proof. Since $h(\beta), h(\gamma)$ and $h\left(1_{H}\right)$ are all $\geqq g(\alpha)$, and $h(\beta) h(\gamma)=g(\alpha)$ by $1.1(12)$ and (3), it is easily seen that $h$ is a lattice homomorphism. Note that $\beta \rightarrow \alpha=\beta \rightarrow \gamma$ $=\gamma$ and $\gamma \rightarrow \alpha=\gamma \rightarrow \beta=\beta$.

If $u<\alpha$, then $h(\beta) \rightarrow h(u)=h(\beta) \rightarrow g(u)=g(u)$, by $1.1(6)$, since $h(\beta) \geqq g(\alpha)$ and $g(\alpha) \rightarrow g(u)=g(\alpha \rightarrow u)=g(u)$ by 3.6. Therefore $h(\beta) \rightarrow h(u)=h(u)=h(\beta \rightarrow u)$ by 1.1(6). A similar argument shows $h(\gamma) \rightarrow h(u)=h(\gamma \rightarrow u)$. Also $h(\beta) \rightarrow h(\alpha)=h(\gamma)$ by 1.1(9), and so $h(\beta) \rightarrow h(\alpha)=h(\beta \rightarrow \alpha)$. Obviously $h(\gamma) \rightarrow h(\alpha)=h(\beta)=h(\gamma \rightarrow \alpha)$. Next $h(\beta) \rightarrow h(\gamma)=h(\gamma)=h(\beta \rightarrow \gamma)$ by $1.1(10)$, and $h(\gamma) \rightarrow h(\beta)=h(\beta)=h(\gamma \rightarrow \beta)$ by 1.1(11). Finally, by using 1.1(7), it is easily seen that $h\left(1_{H}\right) \rightarrow h(\alpha)=h(\alpha)=$ $h\left(1_{H} \rightarrow \alpha\right), \quad h\left(1_{H}\right) \rightarrow h(u)=h(u)=h\left(1_{H} \rightarrow u\right)$ for $u<\alpha, \quad h\left(1_{H}\right) \rightarrow h(\beta)=h\left(1_{H} \rightarrow \beta\right)$, and $h\left(1_{H}\right) \rightarrow h(\gamma)=h\left(1_{H} \rightarrow \gamma\right)$.

THEOREM 3.13. Let $H=\left(\sum_{n<\delta}^{*} H_{n}\right) \oplus 1$, where $0<\delta \leqq \omega$ and, for each $n, H_{n}$ is isomorphic with $B_{2}$ or $B_{4}$. Then $H$ is a projective Heyting algebra. 
Proof. Let $0_{H}=\alpha_{0}<\alpha_{1}<\cdots<\alpha_{\delta}=1_{H}$ be the nodes of $H$. Let

$$
N=\left\{n<\delta: H_{n} \cong B_{4}\right\} \text {. }
$$

For $n \in N, H_{n}=\left\{\alpha_{n}, \beta_{n}, \gamma_{n}, \alpha_{n+1}\right\}$, where $\beta_{n}$ and $\gamma_{n}$ are the atoms of $H_{n}$. For $n \notin N$, $H_{n}=\left\{\alpha_{n}, \alpha_{n+1}\right\}$. Let $F$ be a free Heyting algebra with the set $\left\{x_{n}: 0<n<\delta\right\}$ $\cup\left\{y_{n}: n \in N\right\}$ of free generators. There exists a homomorphism $f: F \rightarrow H$ such that $f\left(x_{n}\right)=\alpha_{n}$ for $0<n<\delta$ and $f\left(y_{n}\right)=\beta_{n}$ for $n \in N$. Clearly $f$ is an epimorphism. We wish to show $H$ is a retract of $F$. For each $n$, let $K_{n}=\bigcup_{i \leqq n} H_{i}$ and $K_{-1}=\left\{0_{H}\right\}$. For $-1 \leqq n<\delta$, define $g_{n}: K_{n} \rightarrow F$ inductively as follows.

Let $g_{-1}\left(0_{H}\right)=0_{F}$. If $n \geqq 0$, let $g_{n}$ be the extension of $g_{n-1}$ such that for $n \notin N$,

and for $n \in N$,

$$
g_{n}\left(\alpha_{n+1}\right)=x_{n+1}+\left(x_{n+1} \rightarrow g_{n-1}\left(\alpha_{n}\right)\right),
$$

$$
\begin{aligned}
g_{n}\left(\beta_{n}\right) & =\left(y_{n} \rightarrow g_{n-1}\left(\alpha_{n}\right)\right) \rightarrow g_{n-1}\left(\alpha_{n}\right) \\
g_{n}\left(\gamma_{n}\right) & =y_{n} \rightarrow g_{n-1}\left(\alpha_{n}\right) \text { and } \\
g_{n}\left(\alpha_{n+1}\right) & =g_{n}\left(\beta_{n}\right)+g_{n}\left(\gamma_{n}\right) .
\end{aligned}
$$

By 3.11 and 3.12, it follows by induction that $g_{n}$ is a pre-homomorphism for $-1 \leqq n<\delta$. Let $g$ be the union (common extension) of all the $g_{n}$ to $\Sigma_{n<\delta}^{*} H_{n}$. Then $g$ is a pre-homomorphism. Extend $g$ to all of $H$ by defining $g\left(1_{H}\right)=1_{F}$. Then $g: H \rightarrow F$ is obviously a homomorphism.

To show $f g=I_{H}$, first note that $f g_{-1}\left(0_{H}\right)=f\left(0_{F}\right)=0_{H}$. Suppose $n \geqq 0$, and we have shown $f g_{n-1}=I_{K_{n-1}}$. If $n \notin N$, then $f g_{n}\left(\alpha_{n+1}\right)=\alpha_{n+1}+\left(\alpha_{n+1} \rightarrow \alpha_{n}\right)=\alpha_{n+1}$. If $n \in N$, then

$$
f g_{n}\left(\beta_{n}\right)=\left(\beta_{n} \rightarrow \alpha_{n}\right) \rightarrow \alpha_{n}=\gamma_{n} \rightarrow \alpha_{n}=\beta_{n}, \quad f g_{n}\left(\gamma_{n}\right)=\beta_{n} \rightarrow \alpha_{n}=\gamma_{n},
$$

and

$$
f g_{n}\left(\alpha_{n+1}\right)=\beta_{n}+\gamma_{n}=\alpha_{n+1} .
$$

Therefore $f g_{n}=I_{K_{n}}$ for all $n<\delta$. Since $f g\left(1_{H}\right)=1_{H}$, it follows that $f g=I_{H}$. Thus $H$ is a retract of a free Heyting algebra, and so $H$ is projective.

\section{Finite projective algebras.}

LemMA 4.1. Suppose $H_{0}$ and $H_{1}$ are Heyting algebras and $H_{0} * H_{1}$ is projective. If $f: A \rightarrow B$ is a Heyting epimorphism such that $f(x)=0$ only for $x=0$, and $h: H_{1} \rightarrow B$ is a homomorphism, then there exists a homomorphism $g: H_{1} \rightarrow A$ such that $f g=h$.

Proof. Let $f^{\prime}: H_{0} * A \rightarrow H_{0} * B$ be defined by $f^{\prime}(x)=x$ for $x \in H_{0}$ and $f^{\prime}(x)$ $=f(x)$ for $x \in A$. Let $h^{\prime}: H_{0} * H_{1} \rightarrow H_{0} * B$ be defined similarly. By 3.9, $f^{\prime}$ and $h^{\prime}$ are homomorphisms and $f^{\prime}$ is an epimorphism. Since $H_{0} * H_{1}$ is projective, there exists a homomorphism $g^{\prime}: H_{0} * H_{1} \rightarrow H_{0} * A$ such that $f^{\prime} g^{\prime}=h^{\prime}$. Since $f^{\prime} g^{\prime}\left(0_{H_{1}}\right)$ $=h^{\prime}\left(0_{H_{1}}\right)=0_{B}$, we have $g^{\prime}\left(0_{H_{1}}\right)=0_{A}$. Hence $g^{\prime}(x) \in A$ for all $x \in H_{1}$. Let $g$ be the restriction of $g^{\prime}$ to $H_{1}$. Then $g: H_{1} \rightarrow A$ is a homomorphism. Clearly $f g(x)=h(x)$ for all $x \in H_{1}$. 
Lemma 4.2. Let $F$ be a filter in a Heyting algebra $H$. Then the following are equivalent:

(1) $F$ is a maximal filter.

(2) The function $f: H \rightarrow B_{2}$ such that $f(x)=1$ if and only if $x \in F$ is a homomorphism.

(3) For each $x \in H$, exactly one of $x, \neg x$ is in $F$. If $a \in H$, then $a$ is an atom if and only if $H^{(a)}$ is a maximal filter.

Proof. This is any easy consequence of [6, Chapter I, 13.10].

LeMmA 4.3. Let $b_{1}, \ldots, b_{n}$ be all the distinct atoms of. a finite Boolean algebra $B$. Let $a_{1}, \ldots, a_{n}$ be atoms (not necessarily distinct) of a Heyting algebra $H$. Then there exists a homomorphism $h: H \rightarrow B$ such that $h(x)=\Sigma\left\{b_{i}: a_{i} \leqq x\right\}$ for all $x \in H$.

Proof. By 4.2 there exist homomorphisms $g_{i}: H \rightarrow B_{2}$ such that $g_{i}(x)=1$ if and only if $x \geqq a_{i}$. Let $g: H \rightarrow\left(B_{2}\right)^{n}$ be the homomorphism defined by $g(x)=$ $\left(g_{1}(x), \ldots, g_{n}(x)\right)$. There exists an isomorphism $f:\left(B_{2}\right)^{n} \rightarrow B$ such that $f\left(e_{i}\right)=b_{i}$, $1 \leqq i \leqq n$, where $e_{i}$ is the element whose $j$ th coordinate is 1 if and only if $j=i$. Then $h=f g$ is the required homomorphism.

Let $F$ be the free Heyting algebra with two free generators $x, y$. Then $R(F)$ is the free Boolean algebra with free generators $\neg \neg x$, $\neg \neg y$. This follows from the fact that the canonical map $\varphi: F \rightarrow R(F)$ is an epimorphism, and the elements $\neg \neg x \neg \neg y, \neg \neg x \neg y, \neg x \neg \neg y$ and $\neg x \neg y$ are all $>0$ (as can be seen by mapping $F$ onto the free Boolean algebra with two free generators). These four elements are the atoms of $R(F)$.

THEOREM 4.4. The subalgebra of $F$ generated by $\neg \neg x \neg \neg y$ and $\neg \neg x \neg y$ is infinite.

Proof. We shall construct a Heyting algebra $H$ containing two elements $x_{1}, y_{1}$ such that the subalgebra generated by $\neg \neg x_{1} \neg \neg y_{1}$ and $\neg \neg x_{1} \neg y_{1}$ is infinite. Let $S$ be an infinite set which is partitioned into a triply infinite sequence of nonempty disjoint sets $a_{n}, b_{n}$ and $c_{n}, 0 \leqq n<\omega$. That is,

and

$$
\begin{array}{ll}
a_{i} \cap b_{j}=a_{i} \cap c_{j}=b_{i} \cap c_{j}=\varnothing & \text { for all } i, j, \\
a_{i} \cap a_{j}=b_{i} \cap b_{j}=c_{i} \cap c_{j}=\varnothing & \text { for } i \neq j,
\end{array}
$$

$$
S=\bigcup_{n<\omega}\left(a_{n} \cup b_{n} \cup c_{n}\right)
$$

Let $x_{0}=a_{0}, y_{0}=b_{0}, z_{0}=c_{0}$, and for each $n<\omega$, let

$$
\begin{aligned}
s_{n} & =\bigcup_{i<n}\left(a_{i} \cup b_{i} \cup c_{i}\right), & & x_{n+1}=a_{n+1} \cup b_{n} \cup c_{n} \cup s_{n}, \\
y_{n+1} & =a_{n} \cup b_{n+1} \cup c_{n} \cup s_{n}, & & z_{n+1}=a_{n} \cup b_{n} \cup c_{n+1} \cup s_{n} .
\end{aligned}
$$

We have

$$
x_{n} \subset x_{m} \text { for } m \geqq n+2, \quad \text { and } \quad x_{n} \subset y_{m}, x_{n} \subset z_{m} \text { for } m \geqq n+1,
$$


as well as similar inequalities obtained by permuting $x, y$ and $z$.

Also for all $n$

$$
x_{n} \cap x_{n+1}=y_{n-1} \cup z_{n-1}, \quad \text { and } \quad x_{n} \cap y_{n}=z_{n-1} \cup z_{n-2}
$$

(with the convention $x_{n}=y_{n}=z_{n}=\varnothing$ for $n<0$ ), and similar equations obtained by permutation. Therefore we may use $\left\{x_{n}: n<\omega\right\} \cup\left\{y_{n}: n<\omega\right\} \cup\left\{z_{n}: n<\omega\right\}$ as a basis for a topology of $S$. Clearly, every infinite union of distinct basic open sets is equal to $S$. Let $H$ be the algebra of all open subsets of $S$. It is not difficult to see that for $n \geqq 0, x_{n} \rightarrow\left(y_{n} \cup z_{n}\right)=x_{n+1}, y_{n} \rightarrow\left(x_{n} \cup z_{n}\right)=y_{n+1}$, and $z_{n} \rightarrow\left(x_{n} \cup y_{n}\right)$ $=z_{n+1}$. Therefore $H$ is generated by $x_{0}, y_{0}$, and $z_{0}$. But $\neg x_{0}=x_{1}, \neg y_{0}=y_{1}$, $\neg z_{0}=z_{1}, \neg x_{1}=x_{0}$, $\neg y_{1}=y_{0}$, and $\neg z_{1}=z_{0}$. Therefore $\neg \neg x_{1} \neg \neg y_{1}=z_{0}$ and $\neg \neg x_{1} \neg y_{1}=y_{0}$, and $y_{0}$ and $z_{0}$ generate $H$ because $x_{0}=\neg y_{0} \neg z_{0}$.

THEOREM 4.5. Suppose $\alpha$ is a node in a projective Heyting algebra $H$. If $\alpha$ has more than two immediate successors, then $H^{(\alpha)}$ is infinite.

Proof. Let $F$ be the free Heyting algebra with two free generators $x, y$. Let $b_{1}=\neg \neg x \neg \neg y, b_{2}=\neg \neg x \neg y, b_{3}=\neg x \neg \neg y$, and $b_{4}=\neg x \neg y$ be the atoms of $R(F)$. Let $a_{1}, a_{2}, a_{3}$ be three distinct atoms of $H^{(\alpha)}$, and let $a_{4}=a_{3}$. By 4.3, there exists a homomorphism $h: H^{(\alpha)} \rightarrow R(F)$ such that $h(u)=\Sigma\left\{b_{i}: u \geqq a_{i}\right\}$. Let $\varphi: F \rightarrow R(F)$ be the canonical epimorphism. By 1.1(8), $\varphi(u)=0$ only for $u=0$. Since $H=H_{(\alpha)} * H^{(\alpha)}, 4.1$ furnishes a homomorphism $g: H^{(\alpha)} \rightarrow F$ such that $\varphi g=h$. Now $g\left(\left(a_{1} \rightarrow \alpha\right) \rightarrow \alpha\right)=\neg \neg g\left(a_{1}\right)=\varphi g\left(a_{1}\right)=h\left(a_{1}\right)=b_{1}$, and similarly $g\left(\left(a_{2} \rightarrow \alpha\right) \rightarrow \alpha\right)=b_{2}$. Therefore $g\left(H^{(\alpha)}\right)$ is infinite by 4.4 . Hence $H^{(\alpha)}$ is infinite.

Lemma 4.6. Let $H$ be a Heyting algebra and $C$ be the three-element chain $\{0, \beta, 1\}$. Then there exists a homomorphism $h: H \rightarrow C$ such that $F_{1}=\{x \in H: h(x) \geqq \beta\}$ and $F_{2}=\{x \in H: h(x)=1\}$ if and only if:

(1) $F_{1}$ is a maximal filter.

(2) $F_{2}$ is a prime filter.

(3) $F_{2} \subseteq F_{1}$.

(4) For all $x \in H$, if $x \notin F_{1}$ then $\neg x \in F_{2}$.

(5) For all $x, y \in H$, if $x, y \in F_{1}-F_{2}$, then $x \rightarrow y \in F_{2}$.

Proof. This is easily checked by straightforward verification.

Lemma 4.7. Suppose $H$ is a finite Heyting algebra, $a$ is an atom of $H, y$ is an immediate successor of $a$, and $a$ is the only atom $\leqq y$. Let $C$ be the three-element chain $\{0, \beta, 1\}$. Then there exists a homomorphism $h: H \rightarrow C$ such that $h(a)=\beta$.

Proof. Let $F_{1}=H^{(a)}$ and $F_{2}=H^{(y)}$. Then $F_{2} \subseteq F_{1}$ and $F_{1}$ is a maximal filter by 4.2. To prove that $F_{2}$ is a prime filter, suppose $u \geq y$. Then $y u=0$ or $y u \geqq$ some atom of $H$. Therefore since $y u<y$, either $y u=0$ or $y u=a$. If $u \geq y$ and $v \geqq y$, but $u+v \geqq y$, then $y=y u+y v \leqq a$, which is a contradiction. To prove condition (4) of 4.6, suppose $x \notin F_{1}$. Then $x y$ cannot be $\geqq$ any atom of $H$. Hence $x y=0$ and so 
$y \leqq \neg x$. For condition (5), suppose $u, v \in F_{1}-F_{2}$. As before, $y u=a$. Hence $y u \leqq v$, which implies $y \leqq u \rightarrow v$. Lemma 4.6 now yields the desired homomorphism.

TheOREM 4.8. If $\alpha$ is a node in a finite projective Heyting algebra $H$, and $\alpha$ has exactly two immediate successors $a_{1}, a_{2}$ then $a_{1}+a_{2}$ is a node.

Proof. Suppose there exists an element $z$ incomparable with $a_{1}+a_{2}$. Since $\alpha$ is a node, we must have $\alpha<z$. Without loss of generality we may assume $a_{1}<z$ and so $a_{2} \$ z$. Let $y$ be an immediate successor of $a_{1}$ such that $y \leqq z$. By 4.7 there exists a homomorphism $h_{1}: H^{(\alpha)} \rightarrow C=\{0, \beta, 1\}$ such that $h_{1}\left(a_{1}\right)=\beta$. By 4.2 there exists a homomorphism $h_{2}: H^{(\alpha)} \rightarrow B_{2}$ such that $h_{2}(\dot{u})=1$ if and only if $u \geqq a_{2}$. Define $h: H^{(\alpha)} \rightarrow C \times B_{2}$ by $h(u)=\left(h_{1}(u), h_{2}(u)\right)$. Let $F$ be the free Heyting algebra with one free generator $x$. Let $f: F \rightarrow C \times B_{2}$ be the homomorphism such that $f(x)=(\beta, 0)$. Since $\neg(\beta, 0)=(0,1)$ and $\neg(0,1)=(1,0),(\beta, 0)$ generates $C \times B_{2}$. Therefore $f$ is an epimorphism. In [5] there is a complete description of $F$. From it we see that the only members of $F$ which are $\$ \neg x+\neg \neg x$ are $0, x, \neg x, x+\neg x$, $\neg \neg x$, and $\neg \neg x \rightarrow x$. Therefore $f(u)=(0,0)$ only for $u=0$, and $f(u)=(\beta, 0)$ only for $u=x$. Therefore by 4.1 , there exists a homomorphism $g: H^{(\alpha)} \rightarrow C \times B_{2}$ such that $f g=h$. Since $f g\left(a_{1}\right)=h\left(a_{1}\right)=(\beta, 0), g\left(a_{1}\right)=x$. But $x$ generates $F$, and $F$ is infinite. Hence $H^{(\alpha)}$ is infinite, which is a contradiction.

THEOREM 4.9. If $H$ is a projective Heyting algebra, then $1_{H}$ is join irreducible.

Proof. The map $f: H \oplus 1 \rightarrow H$ such that $f(1)=1_{H}$ and $f(u)=u$ for $u \in H$ is an epimorphism. Since $H$ is projective, there exists a homomorphism $g: H \rightarrow H \oplus 1$ such that $f g=I_{H}$. Suppose $1_{H}=x+y$. Then $g(x)+g(y)=1$. Since 1 is join irreducible in $H \oplus 1$, either $g(x)=1$ or $g(y)=1$. Therefore either $x=f g(x)=1_{H}$ or $y=1_{H}$.

THEOREM 4.10. If $H$ is a finite Heyting algebra, then $H$ is projective if and only if $H$ is of the form $H=H_{0} * \cdots * H_{n}$, where $H_{n} \cong B_{2}$ and for each $i<n, H_{i} \cong B_{2}$ or $H_{i} \cong B_{4}$.

Proof. The sufficiency was proved in 3.13 for $n>0$ and $B_{2}$ is obviously projective. Suppose $H$ is projective. Let $0=\alpha_{0}<\alpha_{1}<\cdots<\alpha_{n+1}=1$ be the nodes of $H$, and let $H_{i}=\left\{x \in H: \alpha_{i} \leqq x \leqq \alpha_{i+1}\right\}, 0 \leqq i \leqq n$. By 4.5 , each node has at most two immediate successors in $H$. If $\alpha_{i}$ has only one immediate successor $a$, then $a$ is a node and so $a=\alpha_{i+1}$. Therefore $H_{i}=\left\{\alpha_{i}, \alpha_{i+1}\right\}$, which is isomorphic with $B_{2}$. If $\alpha_{i}$ has two immediate successors $a_{1}, a_{2}$, then $\alpha_{i+1} \geqq a_{1}+a_{2}$. Since $a_{1}+a_{2}$ is a node by 4.8 , $a_{1}+a_{2}=\alpha_{i+1}$. Therefore $H_{i}=\left\{\alpha_{i}, a_{1}, a_{2}, \alpha_{i+1}\right\}$, which is isomorphic with $B_{4}$. Finally 4.9 shows that $H_{n}$ cannot be isomorphic with $B_{4}$.

\section{Further results.}

LEMMA 5.1. If $H$ is a projective Heyting algebra, then so are $B_{4} * H$ and $B_{2} * H$.

Proof. Let $\beta$ and $\gamma$ be the atoms of $B_{4}$. Let $\left\{a_{i}: i \in I\right\}$ be the set of members of $H-\left\{0_{H}\right\}$. Let $F$ be a free Heyting algebra with free generating set $\{x\} \cup\left\{x_{i}: i \in I\right\}$. 
There exists a homomorphism $f: F \rightarrow B_{4} * H$ such that $f(x)=\beta$ and $f\left(x_{i}\right)=a_{i}$, $i \in I$. We have $f(\neg x)=\gamma, f(\neg \neg x)=\beta$, and $f(\neg x+\neg \neg x)=\beta+\gamma=0_{H}$. Therefore $f$ is an epimorphism. Let $K=\{u \in F: u \geqq \neg x+\neg \neg x\}$. Let $f_{1}$ be the restriction of $f$ to $K$. Since $f\left(\neg x+\neg \neg x+x_{i}\right)=a_{i}$, and by $3.6 K$ is a Heyting algebra which is a pre-subalgebra of $F, f_{1}: K \rightarrow H$ is an epimorphism. By the projectivity of $H$, there exists a homomorphism $g_{1}: H \rightarrow K$ such that $f_{1} g_{1}=I_{H}$. Let $C_{4}=\left\{0_{F}, \neg x\right.$, $\neg \neg x, \neg x+\neg \neg x$. By 1.1(10), (11) and (7), $C_{4}$ is a pre-subalgebra of $F$. Also the map $g_{0}: B_{4} \rightarrow C_{4}$ such that $g_{0}(0)=0_{F}, g_{0}(\beta)=\neg \neg x, g_{0}(\gamma)=\neg x$ and $g_{0}(1)$ $=\neg x+\neg \neg x$ is an isomorphism. Therefore, by 3.9, the map $g: B_{4} * H \rightarrow C_{4} * K$ defined by $g(u)=g_{1}(u)$ for $u \in H$, and $g(u)=g_{0}(u)$ for $u \in B_{4}$, is a homomorphism. Furthermore $C_{4} * K$ is a subalgebra of $F$, since if $u \geqq \neg x+\neg \neg x, 1.1(5)$ shows that $u \rightarrow \neg \neg x=\neg \neg x, u \rightarrow \neg x=\neg x$ and also $u \rightarrow 0_{F}=0_{F}$. Therefore $g$ may be regarded as a homomorphism $g: B_{4} * H \rightarrow F$. Clearly $f g(u)=u$ for all $u \in B_{4} * H$. Therefore $B_{4} * H$ is a retract of $F$, and so $B_{4} * H$ is projective. The proof of the other half of the lemma is similar. Let $f: F \rightarrow B_{2} * H$ be the epimorphism such that $f(x)=0_{H}$ and $f\left(x_{i}\right)=a_{i}, i \in I$. Let $K=\{u \in F: u \geqq x+\neg x\}$. The restriction $f_{1}$ of $f$ to $K$ is an epimorphism of $K$ onto $H$. There exists a homomorphism $g_{1}: H \rightarrow K$ such that $f_{1} g_{1}=I_{H}$. Let $C_{2}=\left\{0_{F}, x+\neg x\right\}$. As before we can extend $g_{1}$ to a homomorphism $g: B_{2} * H \rightarrow F$ such that $f g(u)=u$ for all $u \in B_{2} * H$.

TheOREM 5.2. If $H_{1}$ is a projective Heyting algebra, and $H_{0}$ is a finite Heyting algebra such that $H_{0} \oplus 1$ is projective, then $H_{0} * H_{1}$ is projective.

Proof. This follows from 5.1 and 4.10 .

Lemma 5.3. Let $H$ be a projective Heyting algebra. Suppose $S$ is an infinite subset of $H$ which is closed under $\rightarrow$. Then $\Sigma(S-\{1\})=1$.

Proof. Suppose the conclusion is false. Then there exists $a \in H$ such that $1>a \geqq u$ for all $u \in S-\{1\}$. Let $f: F \rightarrow H$ be an epimorphism, where $F$ is a free Heyting algebra. There exists a homomorphism $g: H \rightarrow F$ such that $f g=I_{H}$. Let $\alpha=g(a)$. Then $\alpha \neq 1$, since $f(\alpha)=a<1$. Also $\alpha \geqq g(u)$ for all $u \in S-\{1\}$. Since $g$ is a monomorphism, $g(S)$ is infinite and $g(S)$ is closed under $\rightarrow$. By [4, Lemma 2.22], there exists a finite Heyting algebra $K$, and a homomorphism $h: F \rightarrow K$ such that $h(\alpha) \neq 1$. Since $g(S)$ is infinite and $K$ is finite, we have $h(v)=h(w)$ for some $v, w \in g(S)$ such that $v \neq w$. If, say, $v \$ w$, then $v \rightarrow w \neq 1$. Hence $h(\alpha) \geqq h(v \rightarrow w)=h(v) \rightarrow$ $h(w)=1$, which is a contradiction.

THEOREM 5.4. Let $H$ be a chain. Then $H$ is a projective Heyting algebra if and only if $H$ is either finite or has order type $\omega+1$.

Proof. The sufficiency follows from 3.13. Suppose $H$ is projective. Let $a \in H$, $a \neq 1$. Then $H_{(a)} \cup\{1\}$ is closed under $\rightarrow$. By 5.3, $H_{(a)}$ is finite. Since $H_{(a)}$ is finite for all $a<1$, the conclusion follows.

A partial converse of 5.2 is the following. 
THEOREM 5.5. Suppose $H_{0}, H_{1}$ are Heyting algebras such that $H_{0} * H_{1}$ is projective. Then $H_{0}$ is finite and $H_{0} \oplus 1$ is projective.

Proof. Let $S=H_{0} \cup\left\{1_{H_{1}}\right\}$. Then by 3.8 and 5.3, $H_{0}$ is finite. Let $F$ be a maximal filter in $H_{1}$. By 4.2 , there exists a homomorphism $g_{1}: H_{1} \rightarrow B_{2}$ such that $g(u)=1$ if and only if $u \in F$. By 3.9, there exists a homomorphism $g: H_{0} * H_{1} \rightarrow H_{0} * B_{2}$ such that $g(u)=u$ for $u \in H_{0}$ and $g(u)=g_{1}(u)$ for $u \in H_{1}$. Therefore $H_{0} * B_{2}=H_{0} \oplus 1$ is a retract of $H_{0} * H_{1}$, and so $H_{0} \oplus 1$ is projective.

We have not been able to decide whether the projectivity of $H_{0} * H_{1}$ implies that of $H_{1}$.

\section{REFERENCES}

1. G. Birkhoff, Lattice theory, 3rd ed., Amer. Math. Soc. Colloq. Publ., vol. 25, Amer. Math. Soc., Providence, R. I., 1967. MR 37 \#2638.

2. H. B. Curry, Foundations of mathematical logic, McGraw-Hill, New York, 1963. MR 26 \#6036.

3. P. R. Halmos, Lectures on Boolean algebras, Van Nostrand Math. Studies, no. 1, Van Nostrand, Princeton, N. J., 1963. MR 29 \#4713.

4. J. C. C. McKinsey and A. Tarski, On closed elements in closure algebras, Ann. of Math. (2) 47 (1946), 122-162. MR 7, 359.

5. I. Nishimura, On formulas of one variable in intuitionistic propositional calculus, J. Symbolic Logic 25 (1960), 327-331. MR 26 \#25.

6. H. Rasiowa and R. Sikorski, The mathematics of metamathematics, Monogr. Mat., Tom 41, PWN, Warsaw, 1963. MR 29 \#1149.

UNIVERSITY OF MISSOURI-ST. LOUIS,

St. Louis, Missouri 63121

UNIVERSITY OF CALIFORNIA,

Los ANGeles, California 90024 